\title{
Biotransformation of Curcumin to Calebin-A: A Pharmacologically Important Novel Curcuminoid from Curcuma Species
}

\author{
Anju Majeed ${ }^{1,2 *}$, Muhammed Majeed ${ }^{1}$, Nooruddin Thajuddin ${ }^{2}$, Sivakumar \\ Arumugam $^{1}$, Furqan Ali $^{1}$ and Kirankumar Beede ${ }^{1}$
}

${ }^{1}$ Sami Labs Limited, 19/1 \& 19/2, 1st Main, 2nd Phase, Peenya Industrial Area, Bangalore - 560 058, Karnataka, India. ${ }^{2}$ Department of Microbiology, Bharathidasan University, Tiruchirappalli - 620 024, Tamil Nadu, India.

\begin{abstract}
Calebin-A, a novel natural product of turmeric (Curcuma longa) rhizome has been reported to possess various pharmacological activities. The minimal natural occurrence has been the major constraint for exploiting its pharmacological use. Thus, this study focuses to investigate the production of calebin-A through biotransformation approach using curcumin as the substrate. Four bacterial strains (Acinetobacter calcoaceticus delfa L360, Pseudomonas putida TGPNP13, P.putida NCIMB 10007 and A.johnsonii NCIMB 9871) and three different media compositions (MTSB, modified tryptic soy broth; TSB, tryptic soy broth; NB, nutrient broth) were used to investigate the conversion of curcumin to calebin-A. Biotransformation studies were carried out by supplementing curcumin $(50 \mathrm{mg})$ into media $(1000 \mathrm{ml})$. After $24 \mathrm{~h}$ of interval, samples were withdrawn and ethyl acetate extract was obtained as per partition method followed by determination of calebin-A presence using HP-TLC and HPLC techniques. P. putida NCIMB 10007 and A. johnsonii NCIMB 9871 were found to convert curcumin to calebin-A in a time dependent manner with optimum conversion at $48 \mathrm{~h}$ and after $96 \mathrm{~h}$ respectively. The highest growth of $P$. putida NCIMB 10007 and $A$. johnsonii NCIMB 9871 and the production of calebin-A were observed when grown in MTSB media. Baeyer-Villiger monoxygenase enzyme was produced by $P$. putida NCIMB $10007(3.30 \pm 0.09 \mathrm{U} / \mathrm{ml})$ and A. johnsonii NCIMB $9871(4.10 \pm 0.10 \mathrm{U} / \mathrm{ml})$, suggesting its possible role in the conversion of curcumin to calebin-A. The findings of this study clearly suggested that $P$. putida NCIMB 10007 and $A$. johnsonii could be potential candidates for biotechnological production of calebin-A from curcumin.

Keywords: Calebin-A, biotransformation, Baeyer-Villiger monooxygenase, Acinetobacter calcoaceticus, Pseudomonas putida.
\end{abstract}

*Correspondence: anju@samilabs.com; +91 8028397973

(Received: 10 April 2019; accepted: 20 May 2019)

Citation: Anju Majeed, Muhammed Majeed, Nooruddin Thajuddin, Sivakumar Arumugam, Furqan Ali and Kirankumar Beede, Biotransformation of Curcumin to Calebin-A: A Pharmacologically Important Novel Curcuminoid from Curcuma species, J Pure Appl Microbiol., 2019; 13(2): 1159-1168. doi: 10.22207/JPAM.13.2.55

(c) The Author(s) 2019. Open Access. This article is distributed under the terms of the Creative Commons Attribution 4.0 International License which permits unrestricted use, sharing, distribution, and reproduction in any medium, provided you give appropriate credit to the original author(s) and the source, provide a link to the Creative Commons license, and indicate if changes were made. 


\section{INTRODUCTION}

Curcuma longa L. (turmeric) has been known as a color food additive, spice and also used as remedy to treat and manage various human aliments in traditional Asian medicine for centuries (Praditya et al., 2019). Turmeric rhizomes containing active curcumin, has been reported to exhibit several pharmacological properties such as antibacterial, anticoagulation, anti-inflammation, anticancer activities and also help improve blood circulation, lipid metabolism, hormonal disorders and indigestion (Amalraj et al., 2017). Curcumin from turmeric has already been proven non-toxic to human and also found to be clinically effective for the treatment of human cancer and other aliments in various human clinical trials worldwide (Kunnumakkara et al., 2016). Regardless of its various biological activities, curcumin is reported to have low bioavailability, low solubility in water and also strong color intensity which limits its uses in pharmacology (Liu et al., 2016). Thus, various researchers have made efforts to explore new metabolites and curcumin derivatives from turmeric rhizomes which have been reported to show better efficacy and also to have improved other characteristics. The study conducted by Younis et al., (2016) suggested that microbial transformation of curcumin can yield more biologically active metabolites which showed highest anticancer, antimicrobial and anti-oxidant activity than that of curcumin (Amalraj et al., 2017). Furthermore, efforts have been made to increase the bioavailability of curcumin using biotransformation approach through glycosylation reaction which resulted in the production of the compound i.e. curcumin (Purpura et al., 2018). However, limited success has been achieved through all the various approaches to overcome the low bioavailability, low solubility in water and strong color intensity. Thus, there is a need existing to search for an alternative curcuminoid compound with improved efficacy and other characteristics. Calebin-A (3E)-4-(4Hydroxy-3-methoxyphenyl) 2-oxo-3-buten-1-yl (2E)-3-(4-hydroxy-3 metho-xyphenyl) acrylate)) is a curcuminoid reported to be present in the Curcuma species along with other curcuminoids (curcumin) which has a highly similar structural feature as curcumin (Dosoky and Setzer 2018). Calebin-A was first isolated and reported by the
Kim and Kim (2001) from Curcuma longa rhizome with the yield of $0.001 \%(w / w)$ of the total turmeric extract. There are reports suggesting that the calebin-A has promising activity to prevent $\beta$ - amyloid $(\beta A)$ insult to neuronal cells in Alzheimer's disease (Yan et al. 1996; Park and Kim 2002). Among several pharmacological activities, calebin-A has also been reported to show anticancer effects and to improve hepatic steatosis, suppress adipocyte differentiation and prevented high fat diet-induced obesity (Lai et al., 2015). By considering the pharmacological activities exhibited by the calebin-A till date, there is scope that the calebin-A could be a potential agent in the treatment and management of various human ailments including Alzheimer's disease, cancer and obesity. However, the trace quantity reported in turmeric rhizome is a limiting factor for the development of calebin-A. There are reports suggesting the chemical synthesis of calebin-A (Majeed et al. 2017), but obtaining calebin-A naturally is still a challenge to the researchers.

The term biotransformation refers to the organic reactions involving biological catalysts which can either be whole cells or enzymes, in order to chemically modify a chemical compound (Lin and Tao, 2017). Of late, biotransformation approaches have gained a lot of popularity among industries as these processes are environment friendly and products obtained through these processes can also be considered natural if the substrate is from natural origin, according to the FDA and European legislation (Gallage and Miller, 2015). Due to the natural origin of curcumin and also its simple and similar structure as calebin-A, curcumin could be an ideal substrate to obtain calebin-A through biotransformation approach. To the best of our knowledge, there is no report suggesting the bacterial biotransformation of natural curcumin to calebin-A. Thus, the current study aimed to investigate the potential of Acinetobacter johnsonii NCIMB 9871 and Pseudomonas putida NCIMB 10007 for the bioconversion of curcumin to calebin-A.

\section{MATERIALS AND METHODS}

Tryptone soy broth (TSB) and nutrient broth (NB) were purchased from HiMedia Laboratories Pvt. Ltd (Mumbai, India). tetrahydrofuran, mercuric chloride, sodium hypo- 
chlorate, methanol, dimethyl sulfoxide (DMSO), $\beta$-nicotinamide adenine dinucleotide 22 -phosphate, reduced tetrasodium salt hydrate (NADPH) and cyclohexanone were procured from Sigma-Aldrich (St. Louis, MO, USA). TLC Silica Gel 60 F254 was procured from Merck KGaA (Darmstadt, Germany). Curcumin and calebin-A were obtained from Sabinsa Corporation (Piscataway, NJ, USA) and were used as standard throughout the study. Bacterial strains $A$. calcoaceticus delfa L360 and $P$. putida TGPNP13 were procured from National Centre for Cell Science (NCCS), Pune, India. $P$. putida NCIMB 10007 and A. johnsonii NCIMB 9871 were obtained from NCIMB Ltd, Aberdeen, Scotland.

Screening of microbes for the growth and biotransformation ability

The screening of bacteria for the potential to biotransformation of curcumin to calebin $A$ was performed. Different bacterial strains i.e. $A$. calcoaceticus delfa L360, $P$. putida, TGPNP13 $P$. putida NCIMB 10007 and A. johnsonii NCIMB 9871 were screened by growing in different media i.e. MTSB (pancreatic digest of casein $17 \mathrm{~g} / \mathrm{L}$, papaic digest of soyabean meal $3 \mathrm{~g} / \mathrm{L}$, sodium chloride 5 $\mathrm{g} / \mathrm{L}$, dextrose $5 \mathrm{~g} / \mathrm{L}$, dibasic potassium phosphate $2.5 \mathrm{~g} / \mathrm{L}$, manganese(II) sulfate $0.1 \mathrm{~g} / \mathrm{L}$, magnesium sulfate $\mathrm{g} / \mathrm{L}$, calcium chloride $0.1 \mathrm{~g} / \mathrm{L}$, potassium dihydrogen phosphate $1 \mathrm{~g} / \mathrm{L} ; \mathrm{pH} 7.0 \pm 2$ ), TSB (pancreatic digest of casein $17 \mathrm{~g} / \mathrm{L}$, papaic digest of soyabean meal $3 \mathrm{~g} / \mathrm{L}$, sodium chloride $5 \mathrm{~g} / \mathrm{L}$, dextrose $2.5 \mathrm{~g} / \mathrm{L}$, dibasic potassium phosphate $2.5 \mathrm{~g} / \mathrm{L}, \mathrm{pH} 7.3 \pm 2$ ), NB (peptone $5 \mathrm{~g} / \mathrm{L}$, sodium chloride $5 \mathrm{~g} / \mathrm{L}$, HM peptone $1.5 \mathrm{~g} / \mathrm{L}$, yeast extract $1.5 \mathrm{~g} / \mathrm{L} ; \mathrm{pH} 7.3 \pm 2$ ). Overnight grown cultures of $P$. putida NCIMB 10007 and A. johnsonii NCIMB 9871 were inoculated in to MTSB, TSB and NB media. After every $24 \mathrm{~h}$ of time intervals, samples were collected and analyzed for total reducing sugars (TRS) as described earlier and optical density (OD) was recorded by using a UV-VIS spectrophotometer (Shimadzu Corporation, Kyoto, Japan).

\section{Biotransformation of Curcumin into Calebin-A}

Overnight grown culture of $P$. putida NCIMB 10007 and A. johnsonii NCIMB 9871 were inoculated to MTSB media $(1000 \mathrm{ml})$ supplemented with curcumin $(50 \mathrm{mg}$ dissolved in $20 \mathrm{ml}$ of DMSO) and then flasks were incubated at $37^{\circ} \mathrm{C}$ for $144 \mathrm{~h}$ in shaker incubator (Scigenics Biotech, Chennai, India) with $140 \mathrm{rpm}$. At every $24 \mathrm{~h}$ interval, samples were withdrawn $(100 \mathrm{ml})$ and extracted using ethyl acetate $(300 \mathrm{ml})$ in a separating funnel (Borosil, Mumbai. India). The organic layer was collected and concentrated to dryness using rotary evaporator (Heidolph, Schwabach, Germany). Samples were further dissolved in $20 \mathrm{ml}$ of methanol and this sample was used to identify and for quantitative and qualitative analysis of curcumin and calebin-A.

\section{High Performance Thin Layer Chromatography} (HPTLC)

Qualitative identification of curcumin and calebin-A was performed by using HPTLC system (Camag, Muttens, Switzerland) using silica gel plates $60 \mathrm{~F}_{254^{\prime}}, 20 \times 10 \mathrm{~cm}$ (Merck Millipore) developed by the solvent system chloroform:

Table 1. The utilization of reducing sugar (g/L) by the A. johnsonii NCIMB 9871 and P. putida NCIMB 10007 in three different media compositions determined by following DNSA method

\begin{tabular}{|c|c|c|c|c|c|c|c|}
\hline \multirow[b]{2}{*}{ S.N. } & \multirow[b]{2}{*}{ Time (h) } & \multicolumn{3}{|c|}{ A. johnsonii NCIMB 9871} & \multicolumn{3}{|c|}{ P. putida NCIMB 10007} \\
\hline & & MTSB & TSB & NB & MTSB & TSB & NB \\
\hline 1 & 0 & $3.51 \pm 0.09$ & $2.81 \pm 0.09$ & $0.72 \pm 0.02$ & $3.53 \pm 0.09$ & $2.82 \pm 0.08$ & $0.74 \pm 0.08$ \\
\hline 2 & 24 & $1.91 \pm 0.07$ & $1.34 \pm 0.08$ & $0.43 \pm 0.01$ & $0.60 \pm 0.05$ & $1.45 \pm 0.06$ & $0.45 \pm 0.07$ \\
\hline 3 & 48 & $0.51 \pm 0.04$ & $0.67 \pm 0.06$ & $0.39 \pm 0.01$ & $0.52 \pm 0.04$ & $0.51 \pm 0.05$ & $0.42 \pm 0.05$ \\
\hline 4 & 72 & $0.48 \pm 0.02$ & $0.47 \pm 0.06$ & $0.38 \pm 0.01$ & $0.47 \pm 0.03$ & $0.43 \pm 0.04$ & $0.43 \pm 0.04$ \\
\hline 5 & 96 & $0.45 \pm 0.02$ & $0.45 \pm 0.05$ & $0.39 \pm 0.01$ & $0.45 \pm 0.02$ & $0.44 \pm 0.02$ & $0.42 \pm 0.02$ \\
\hline 6 & 120 & $0.43 \pm 0.02$ & $0.41 \pm 0.04$ & $0.37 \pm 0.01$ & $0.43 \pm 0.01$ & $0.43 \pm 0.01$ & $0.41 \pm 0.01$ \\
\hline 7 & 144 & $0.41 \pm 0.01$ & $0.39 \pm 0.01$ & $0.38 \pm 0.01$ & $0.42 \pm 0.01$ & $0.41 \pm 0.01$ & $0.39 \pm 0.01$ \\
\hline
\end{tabular}

MTSB, modified tryptic soy broth; TSB, tryptic soy broth; NB, Nutrient Broth.

Data are expressed as mean \pm standard deviation (SD) of two independent experiments performed in triplicate. 
methanol (98:2). Each sample $(2 \mu \mathrm{l})$ was loaded with Camag Linomate $V$ semi-automatic sample applicator, Linomat syringe $(100 \mu \mathrm{l})$, with $10 \mathrm{~mm}$ band width. The plates scanned at $280 \mathrm{~nm}$ with deuterium illumination using scanner 3 (Camag). The images were captured on Camag reprostar 3 with win CATS software (ver. 1.4.3.6336) and compared the $R_{f}$ value with the standard of calebin-A.

\section{High Performance -Liquid Chromatography (HPLC)}

The quantification of curcumin and calebin-A was performed by using HPLC (Shimadzu Class Vp series) which was coupled to an auto sampler and DAD-UV detector (SPD-M10A Vp),
C18 column $(250 \times 4.6 \mathrm{~mm})$ and binary gradient pump (LC20 AD). The injection volume was $20 \mu$. The mobile phase used was tetra-hydrofuran $0.6 \%$ citric acid in water (40:60) at a flow rate of $1.0 \mathrm{ml} /$ min. Data acquisition and analysis was carried out using Shimadzu LC Solution version 1.25. Samples were taken in triplicates for the analysis.

Baeyer Villiger Monooxygenases (BVMO) enzyme activity

The overnight grown culture of $P$. putida NCIMB 10007 and A. johnsonii NCIMB 9871 was inoculated into the MTSB, TSB and NB media containing $0.05 \mathrm{mg} / \mathrm{ml}$ of curcumin (dissolved in $2.5 \mathrm{mg} / \mathrm{ml}$ of DMSO) and incubated for $144 \mathrm{~h}$ at

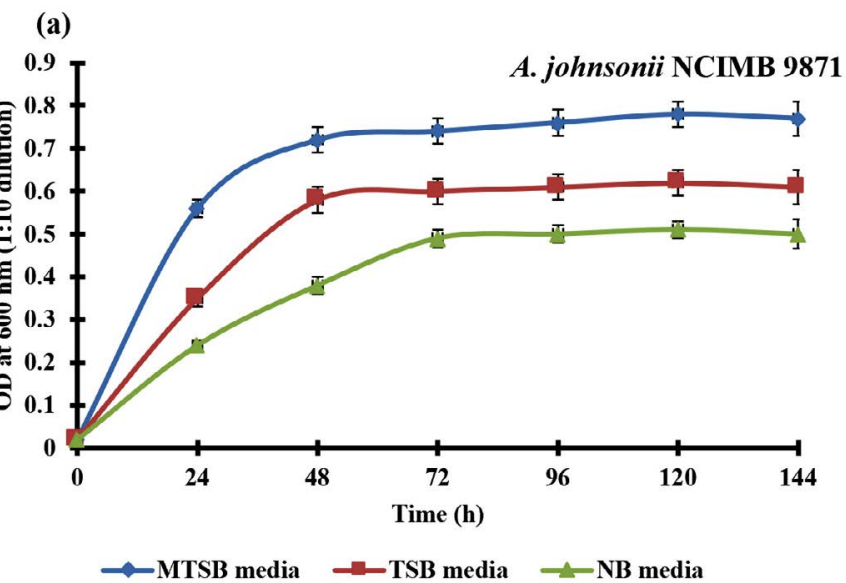

(b)

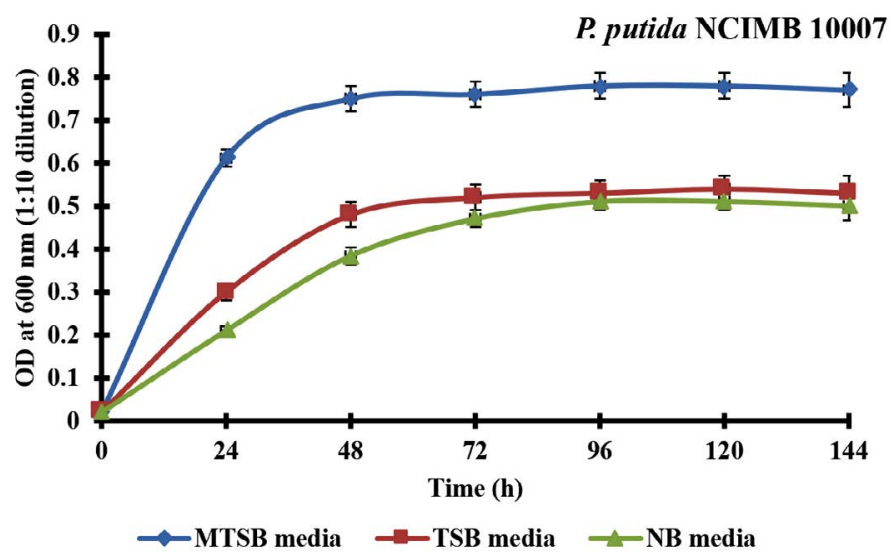

Fig. 1. Growth curve of bacterial strains (a) A. johnsonii NCIMB 9871 and (b) P. putida NCIMB 10007 in three different media compositions. Data are expressed as mean \pm standard deviation (SD) calculated over two independent experiments performed in triplicate. 
$37 \pm 0.5^{\circ} \mathrm{C}$ at $140 \mathrm{rpm}$. After every $24 \mathrm{~h}$, culture was collected and centrifuged $(10,000 \times \mathrm{g}$ for 10 $\mathrm{min}$ ) to remove cells and then supernatant was collected separately. The cell pellet was washed three times with sterile phosphate buffer $(0.1 \mathrm{M}$ PBS, $\mathrm{pH}$ 7.5). The washed cells were re-suspended in $10 \mathrm{ml}$ phosphate buffer (50 mM; $\mathrm{pH} 7.5)$, and then were sonicated by (30 seconds each cycle, with gap of 3 min after each cycle) using ultrasonic homogenizer (Sartorius AG, Gottingen, Germany). The cell homogenate was centrifuged
$(10,000 \times \mathrm{g})$ at $4^{\circ} \mathrm{C}$ and supernatant used as crude enzyme for the BVMOs activity. The BVMOs assay were performed in tris- $\mathrm{HCl}$ buffer $(50 \mathrm{mM}, \mathrm{pH}$ 8.5) containing $10 \mathrm{mM}$ cyclohexanone, $0.8 \mathrm{mM}$ $\mathrm{NADPH}$, by adjusting absorbance between 0.9 to 1.0 at $340 \mathrm{~nm}$ using UV-VIS spectrophotometer (Shimadzu Corporation, Kyoto, Japan). Enzyme activity was measured by monitoring the NADPH consumption at $340 \mathrm{~nm}$ for 180 seconds. One unit (U) of the enzyme activity was defined as the amount of enzyme to oxidize $1 \mu \mathrm{mol}$ of NADPH for

(a)

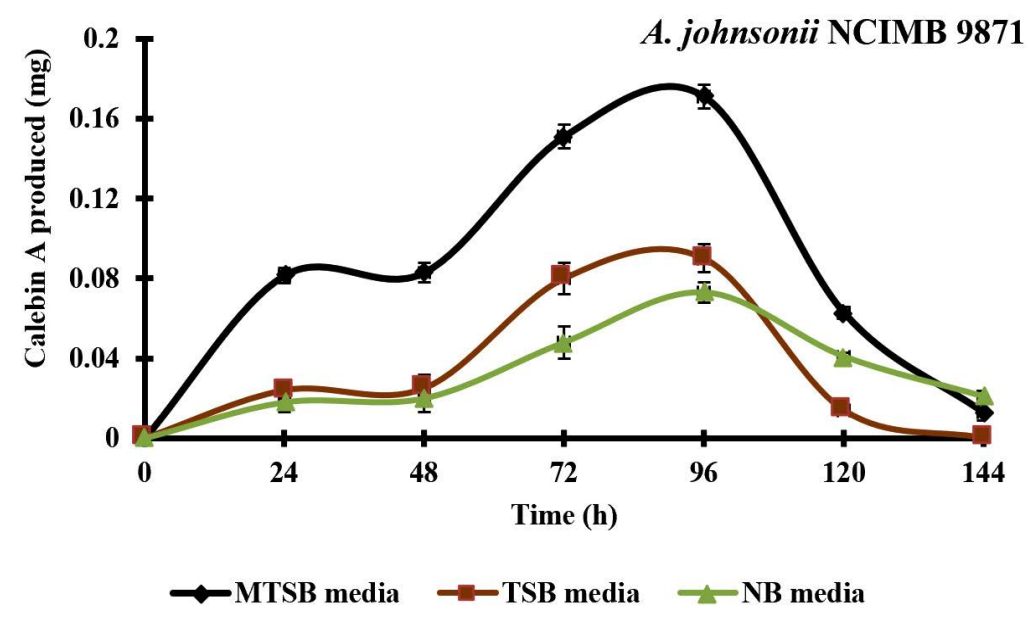

(b)

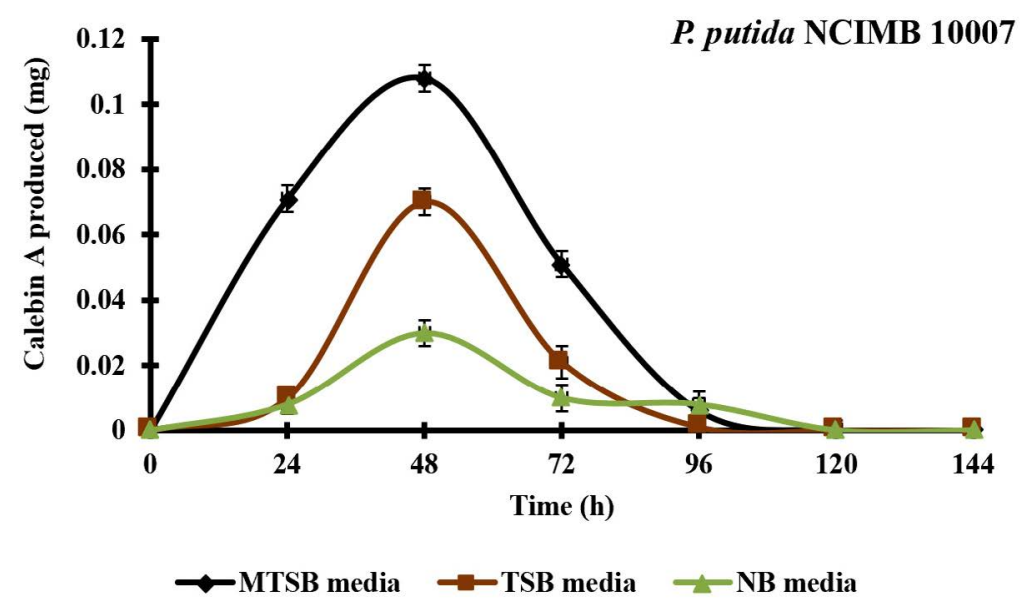

Fig. 2. Effect of different media composition on the production of calebin-A by the A. johnsonii NCIMB 9871 (a) and $P$. putida NCIMB 10007 (b) as determined by the HPLC method. Each value represents the mean \pm SD $(n=3)$. 
$1 \mathrm{~min}$ under the reaction conditions. Estimation of protein was determined as per the Bradford's method as described earlier (Bradford, 1976).

\section{RESULTS}

\section{Growth profile of bacterial strains}

The growth profile of bacterial strains was determined using different media composition (without the supplementation of curcumin). Bacterial strains P. putida NCIMB 10007 and $A$. johnsonii NCIMB 9871 achieved maximum growth at $48 \mathrm{~h}$ in the MTSB media with optical density of 0.75 and 0.72 ( $1: 10$ dilution) at $600 \mathrm{~nm}$ respectively
(Fig. 1a \& b) which suggested that growth of these bacterial strains was higher in MTSB media compared to other media compositions (TSB and NB) (Fig. 1a \& b). The total reducing sugar (TRS) was also analyzed to understand the growth of bacteria. We found that there was decrease in TRS with the increase in growth irrespective of the media used for the growth of $P$. putida NCIMB 10007 and $A$. johnsonii NCIMB 9871 (Table 1). However, the reduction of reducing sugar by the $P$. putida NCIMB 10007 and A. johnsonii NCIMB 9871 was highest in MTSB media composition compared to TSB and NB. (a)

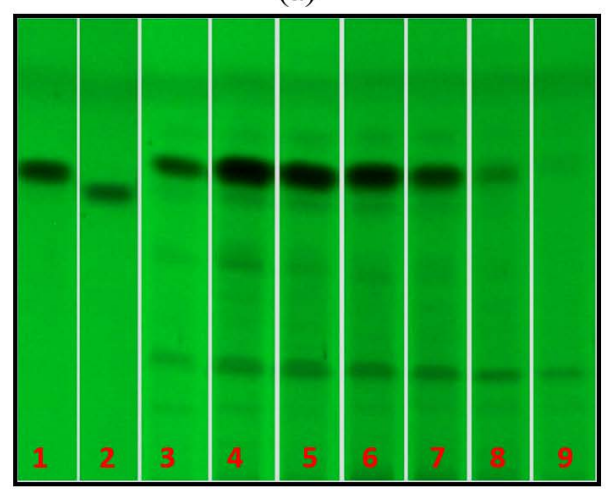

(b)

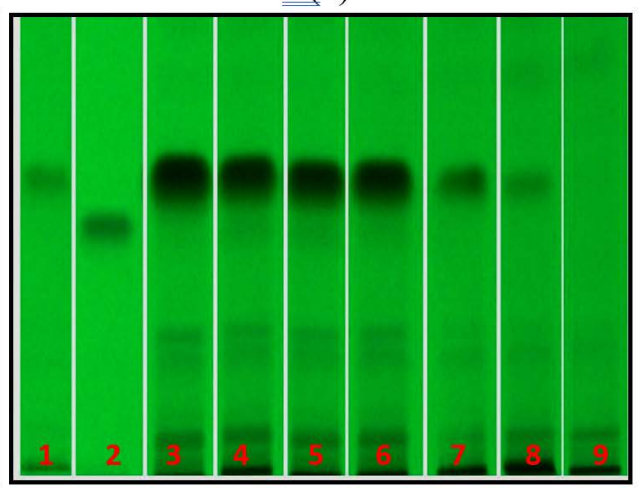

Fig. 3. HPTLC Chromatograms of ethyl acetate extract of (a) A. johnsonii NCIMB 9871 and (b) P. putida NCIMB 10007 at different time intervals of incubation. Lane 1 and 2 represent the curcumin and calebin-A standards in each chromatogram. Lanes 3 to 9 represent the ethyl acetate extract of (a) A. johnsonii NCIMB 9871 and (b) P. putida NCIMB 10007 after 0, 24, 48, 72, 96, 120 and $144 \mathrm{~h}$ of incubation.
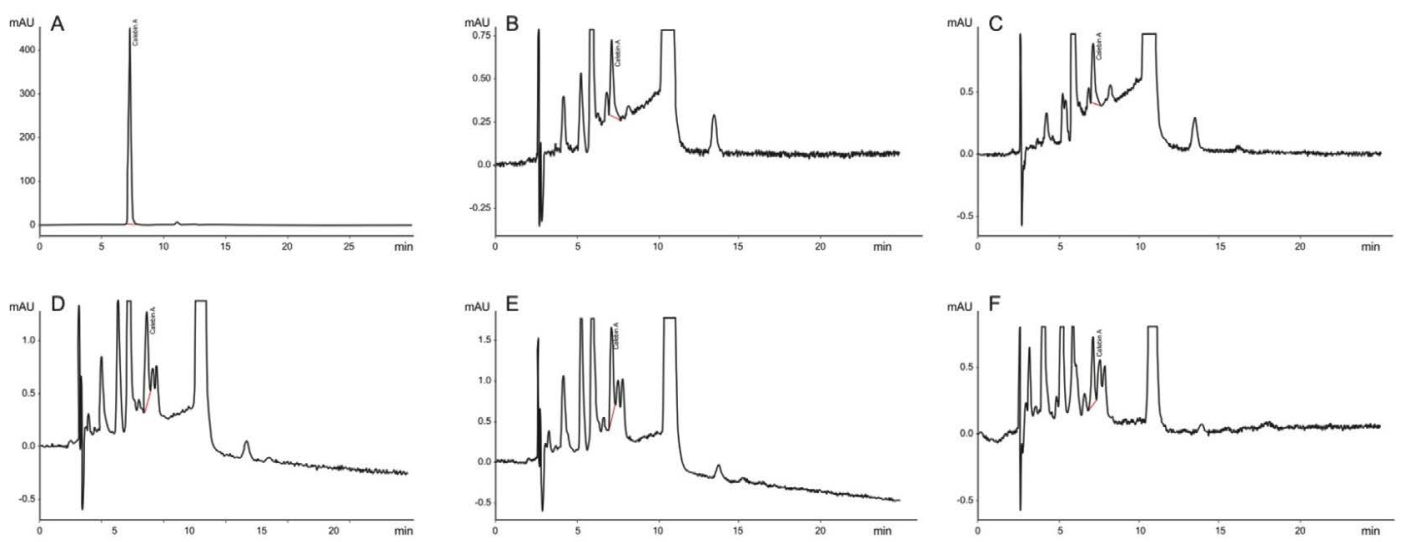

Fig. 4. HPLC chromatogram of $A$. johnsonii NCIMB 9871 at different time intervals of growth and extracted with ethyl acetate (A) Calebin-A standard; (B), 24 hours of growth; (C), 48 hours of growth; (D), 72 hours of growth; (E), 96 hours of growth; (F), 120 hours of growth. The chromatogram shows the presence of calebin-A from the 24 hours of growth after adding curcumin in the broth. 


\section{Screening of microbes for the bioconversion of curcumin to calebin-A}

Four different bacterial strains were screened for the bioconversion of curcumin to calebin-A using three different media composition, aiming to investigate the suitable media and strain for the conversion of curcumin to calebin-A. Data of the experiments revealed that the bioconversion of curcumin to calebin-A was achieved by $P$. putida NCIMB 10007 and $A$. johnsonii NCIMB 9871 in all three media composition (MTSB, TSB and NB). P. putida NCIMB 10007 and $A$. johnsonii NCIMB 9871 were fermented in MTSB, TSB and NB media supplemented with curcumin. The ethyl acetate extract was analyzed for the presence of calebin-A by following HPTLC, HPLC techniques. The HP-TLC results showed the similar band to the calebin- $A$ standard with the same $\mathrm{Rf}$ value. This indicated that the P. putida NCIMB 10007 and A. johnsonii NCIMB 9871 were able to convert the curcumin into calebin-A in the suitable growth time and conditions provided (Fig. 3). Furthermore, quantitative analysis of bio-conversion of curcumin to calebin-A was confirmed by HPLC technique.
The HPLC chromato-gram confirmed the presence of calebin-A in samples as it showed similar retention time as standard of calebin-A (Fig. 4 \& 5). The HPLC chromatogram showed that the primary metabolite and calebin-A appeared after incubation time of $24 \mathrm{~h}$ with retention time (RT) of 7.1 in the $A$. johnsonii NCIMB 9871 and P. putida NCIMB 10007 group (Fig. 4 \& 5). The maximum conversion of calebin-A was observed at $96 \mathrm{~h}$ and $48 \mathrm{~h}$ in the $A$. johnsonii NCIMB 9871 and $P$. putida NCIMB 10007 when grown in MTSB media respectively (Fig. 2a \& b). However, the production of calebin-A by the $A$. johnsonii NCIMB 9871 was significantly higher compared to $P$. putida NCIMB 10007 (Fig. 2a \& b), thereby suggesting better efficiency of bioconversion of curcumin to calebin-A

\section{BVMO enzyme assay}

BVMOs are widely known for oxidization of heteroatom-containing molecules and also to catalyze the insertion of an oxygen atom in ketones and aldehydes. In this study, we have evaluated the intracellular enzyme activity of $P$. putida NCIMB 10007 and A. johnsonii NCIMB 9871
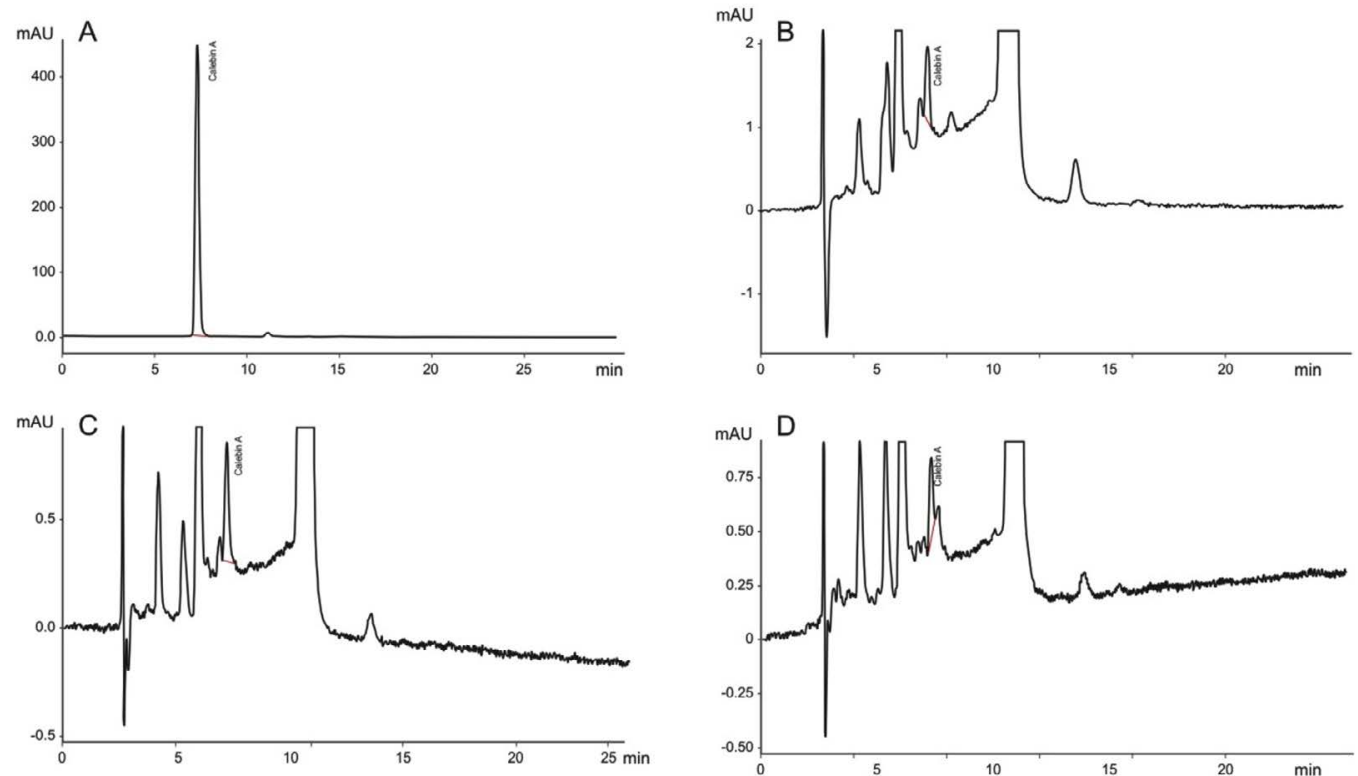

Fig. 5. HPLC chromatogram of $P$. putida NCIMB 10007 at different time intervals of growth and extracted with ethyl acetate (A) Calebin-A standard; (B), 24 hours of growth; (C), 48 hours of growth; (D), 72 hours of growth; (E), 96 hours of growth; (F), 120 hours of growth. The chromatogram shows the presence of calebin-A from the 24 hours of growth after adding curcumin in the broth. 
during the bioconversion of curcumin to calebin-A, in order to investigate the possible role of BVMOs for this conversion. P. putida NCIMB 10007 and $A$. johnsonii NCIMB 9871 produced significant BVMOs enzyme with maximum activity of $3.30 \pm 0.09 \mathrm{U} / \mathrm{ml}$ and $4.10 \pm 0.10 \mathrm{U} / \mathrm{ml}$ respectively (Fig. 6). Data of the study indicated that the pattern of the specific activity was exactly similar with the bioconversion of curcumin to calebin-A by P. putida NCIMB 10007 and $A$. johnsonii NCIMB 9871, thereby indicating BVMOs role in this bioconversion.

(a)

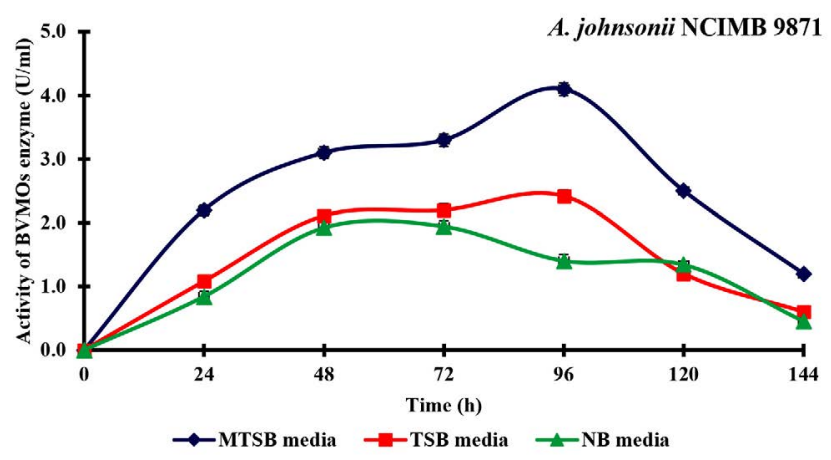

(b)

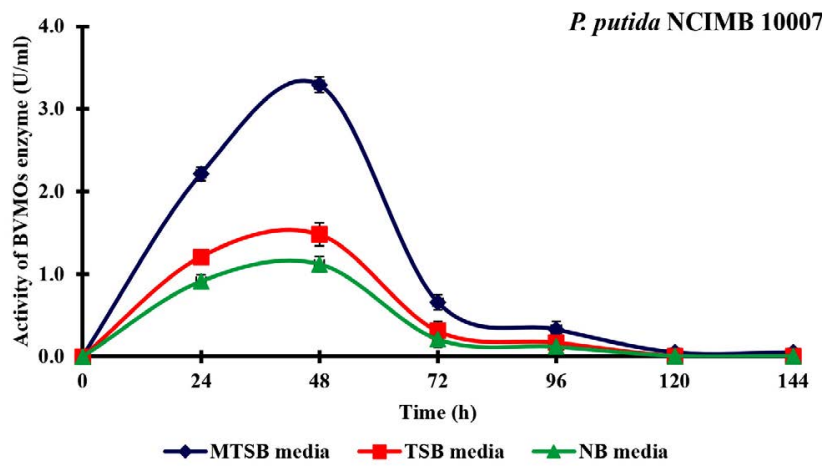

Fig. 6. Effect of different media compositions supplemented with curcumin on the production of BVMOs enzyme by the (a) A. johnsonii NCIMB 9871 and (b) P. putida NCIMB 10007 at different time intervals. Data represents mean \pm standard deviation of three independent experiments.

\section{DISCUSSION}

This study reported for the first time the bioconversion of curcumin to calebin-A aiming to obtain calebin-A naturally apart from its natural occurrence which was first time reported by Kim and Kim (2001) in C. longa rhizome. Previous studies suggested that the calebin-A was found to be a pharmacologically active compound to protect cells from beta-amyloid insult and also effective in the treatment of multidrug resistance cancer and human gastric (Kim and Kim, 2001; Li et al., 2008). Furthermore, calebin-A also reported for higher ability to inhibit the proliferation of HepG2 cells and anti-obesity potential (Chen et al. 2009). In view of these studies, to achieve maximum growth in order to get maximum bioconversion of curcumin to calebin-A conditions for the growth of culture are important. The conditions for the growth of the culture, initial $\mathrm{pH}$, temperature and media play a pivotal role in the bioconversion (Xiao-kui Ma and Daugulis, 2014). Moreover, previous report on curcumin glucoxilation by Catharanthus roseus cells in liquid culture suggested that the glucoxilation efficiency was dependent on the culture growth and sucrose concentration (Kaminaga et al., 2003). Moreover, literature suggested that the biotransformation process have strong association with the growth 
of microbes and enzyme production during the fermentation which depend on the availability of nutrients to the microbes (Singh et al., 2017). In the present study, MTSB media composition was the most suited media to achieve the maximum growth of $P$. putida NCIMB 10007 and $A$. johnsonii $\mathrm{NCIMB} 9871$ and to attain maximum bioconversion of curcumin to calebin-A (Fig. $1 \& 2$ ). These results indicated that MTSB media fulfilled all requirement to grow and produce enzymes which was required for the bio-conversion of curcumin to calebin-A. Interestingly, out of four strains two strains successfully transformed curcumin to calebin-A while growing in MTSB media, suggesting that the failure of biotransformation was not only dependent on the media but it also involved type of culture used in the study.

To obtain pharmacologically active and novel structurally analog compounds, microbial biotransformation has emerged as an important tool (Bianchini et al., 2015). Events in the biotransformation reaction are challenging to get stable, pharmacologic active and non toxic compound, although it has become quite promising due to the availability of large diversity of microbes (Fura, 2006). Pseudomonas putida NCIMB 10007 and A. johnsonii NCIMB 9871 were widely studied bacteria for the camphor and cyclohexanone metabolism (Donoghue 1976; Grogan et al., 1993). Thus, the current study investigated the role of $P$. putida NCIMB 10007 and $A$. johnsonii NCIMB 9871 in the production of calebin-A using curcumin as a substrate. The production of calebin-A was also confirmed by the HP-TLC and HPLC techniques. The pattern of change in composition of the curcumin and calebin-A in the HPLC chromatogram revealed that the P. putida NCIMB 10007 and A. johnsonii NCIMB 9871 were able to convert the curcumin into calebin-A with the highest conversion after 48 and $96 \mathrm{~h}$ of incubation period respectively (Fig. 2). Although this study is preliminary and required further optimization and scale-up in order to develop a economical commercial process to obtain natural calebin- $A$, this is the first report to illustrate the production of natural calebin-A through bacterial biotransformation.

$$
\text { Baeyer-Villiger monooxygenases }
$$

(BVMOs) are flavoenzymes, play a key role in the degradation of ketones, steroid and metabolism of terpenoids, cyclic alcohols and alkanes (Tolmie et al., 2018). The rationale for the selection of $P$. putida NCIMB 10007 and A. johnsonii NCIMB 9871 for the bioconversion of curcumin to calebin-A was due to the presence of BVMOs enzyme during the metabolism, as reported earlier (Kadow et al., 2011). The current results also verified the earlier reports that $P$. putida NCIMB 10007 and A. johnsonii NCIMB 9871 can produce BVMOs enzyme even when supplemented with curcumin. The production of calebin-A and the BVMOs enzyme suggesting that the BVMOs could possibly be responsible for this bioconversion. However, further studies may be needed to elucidate the exact mechanism of action for this bio-conversion.

This study reported for the first time bioconversion of curcumin to calebin-A by $P$. putida NCIMB 10007 and A. johnsonii NCIMB 9871 when grown in MTSB media. Furthermore, the conversion of curcumin to calebin-A by the $A$. johnsonii NCIMB 9871 was higher compared to P. putida NCIMB 10007 (Fig. 2). Although further scale-up studies are needed in order to develop a commercially economical process to produce calebin-A naturally, this study has provided lab scale evidence to produce calebin-A which will be very essential information for the future studies.

\section{ACKNOWLEDGEMENTS}

The data described in the current study partly form the basis for patent applications (US 16281120). We also recognize the intellectual property rights for Curcumin C3 Complex ${ }^{\circledR}$ used in the current study. CURCUMIN C 3 COMPLEX ${ }^{\circledR}$ is a registered logo (U.S Trademark Registration No. 4092057) of Sabinsa Corporation, 20 Lake Drive, East Windsor, NJ, USA 08520.

\section{CONFLICTS OF INTEREST}

The authors declare that there is no conflict of interest.

\section{AUTHORS' CONTRIBUTION}

All authors have made substantial, direct and intellectual contribution to the work and approved it for publication. 


\section{FUNDING}

None.

\section{DATA AVAILABILITY}

All datasets generated or analyzed during this study are included in the manuscript.

\section{ETHICS STATEMENT}

This article does not contain any studies with human participants or animals performed by any of the authors.

\section{REFERENCES}

1. Amalraj A., Pius A., Gopi S., Gopi S. Biological activities of curcuminoids, other biomolecules from turmeric and their derivatives - A review. J. Tradit. Complement Med., 2017; 7(2): 205-233.

2. Bianchini L.F., Arruda M.F.C., Vieira S.R., Campelo P.M.S., Gregio AMT, Rosa E.A.R. Microbial biotransformation to obtain new antifungals. Front Microbiol., 2015; 6: 1433.

3. Bradford M.M. A Rapid and sensitive method for the quantification of microgram quantities of protein utilizing the principle of protein-dye binding. Anal. Bioche., 1976; 72 (1-2): 248-254.

4. Chen L., Li S., Liu C.H., Guo H., Li Y. Comparison of inhibitive effects of curcumin and calebin-A on human hepatoma cell line HepG2. J. Fourth Military Medical University, 2009; 30(1): 7-10.

5. Donoghue N.A., Norris D.R., Trudgill P.W. The purification and properties of cyclohexanone oxygenase from Nocardia globerula CL1 and Acinetobacter NCIB 9871. Etir. J. Biochem.,1976; 63:175-192.

6. Dosoky N.S., Setzer W.N. Chemical composition and biological activities of essential oils of Curcuma species. Nutrients, 2018; 10: 1196.

7. Fura A. Role of pharmacologically active metabolites in drug discovery and development. Drug Discov. Today, 2006; 11(3-4): 133-142.

8. Gallage N.J., Miller B.L. Vanillin-bioconversion and bioengineering of the most popular plant flavor and Its De-novo biosynthesis in the vanilla orchid. Mol. Plant, 2015; 8(1): 40-57.

9. Grogan G., Roberts S., Wan P., Willetts A. Camphorgrown Pseudomonas putida, a multi-functional biocatalyst for undertaking Baeyer-Villiger monooxygenase-dependent biotrans-formations. Biotechnol Lett, 1993; 15(9): 913-918.

10. Kadow M., Stefan S., Schmidt M., Bornscheuer U.T. Recombinant expression and purification of the 2,5-diketocamphane 1,2-monooxygenase from the camphor metabolizing Pseudomonas putida strain NCIMB 10007. AMB Express, 2011; 1: 13.

11. Kaminaga Y., Nagatsu A., Akiyama T., Sugimoto N., Yamazaki T., Maitani T., Mizukami H. Production of unnatural glucosides of curcumin with drastically enhanced water solubility by cell suspension cultures of Catharanthus roseus. FEBS Lett., 2003; 555(2): 311-316.
12. Kim D.S., Kim J.Y. Total synthesis of calebin-A, preparation of its analogues, and their neuronal cell protectivity against -amyloid insult. Bioorg. Med. Chem. Lett., 2001; 11: 2541-2543.

13. Kunnumakkara A.B., Bordoloi D., Padmavathi G., Javadi M., Roy N.K., Sahdeo P., Aggarwal B.B. Curcumin, the golden nutraceutical: multi-targeting for multiple chronic diseases. Br. J. Pharmacol., 2017; 174(11): 1325-1348.

14. Lai C.S., Liao S.N., Tsai M.L., Kalyanam N., Majeed M., Majeed A., Ho C.T., Pan M.H. Calebin-A inhibits adipogenesis and hepatic steatosis in high-fat dietinduced obesity via activation of AMPK signaling. Mol. Nutr. Food Res., 2015; 59(10): 1883-1895.

15. Li Y., Li S., Han Y., Liu J., Zhang J., Li F., Wang Y, Liu X., Yao L. Calebin-Ainduces apoptosis and modulates MAPK family activity in drug resistant human gastric cells, Eur. J. Pharmacol., 2008; 591: 252-258.

16. Lin B., Tao Y. Whole-cell biocatalysts by design. Microb. Cell Fact, 2017; 16: 106.

17. Liu W., Zhai Y., Heng X., Che F.Y., Chen W., Sun D., Zhai G. Oral bioavailability of curcumin: problems and advancements. J. Drug Target, 2016; 24(8): 694-702. 18. Majeed A, Nagabhushanam K, Majeed M, Thomas SM, Thajuddin N An expeditious, green and protecting group-free synthesis of calebin A and analogs. Synopen., 2017; 1:125-128.

19. Park S.Y., Kim D.S. Discovery of natural products from Curcuma longa that protect cells from beta-amyloid insult: A drug discovery effort against Alzheimer's disease. J. Natural Products, 65(9): 1227-1231

20. Praditya D., Kirchhoff L., Br ning J., Rachmawati H., Steinmann J., Steinmann E. Anti-infective properties of the golden spice curcumin. Front Microbiol, 2019.

21. Purpura M., Lowery R.P., Wilson J.M., Mannan H., M nch G., Razmovski-Naumovski V., Analysis of different innovative formulations of curcumin for improved relative oral bioavailability in human subjects. Eur. J. Nutr., 2018; 57(3): 929-938.

22. Singh V., Haque S., Niwas R., Srivastava A., Pasupuleti M., Tripathi C.K.M. Strategies for fermentation medium optimization: An in-depth. Front Microbiol., 2016; 7: 2087.

23. Tolmie C., Smit M.S., Opperman D.J. Native roles of Baeyer-Villiger monooxygenases in the microbial metabolism of natural compounds. Nat. Prod. Rep., 2018; 36(2)

24. Xiao-kui Ma, Daugulis A.J. Effect of bioconversion conditions on vanillin production by Amyco-latopsis sp. ATCC 39116 through an analysis of competing byproduct formation. Bioprocess Biosyst. Eng., 2014; 37: 891-899.

25. Yan S.S., Chen D., Yan S., Guo L., Du H., Chen J.X. RAGE is a key cellular target for a beta-induced perturbation in Alzheimer's disease. Front Biosci., (Schol Ed). 2012; 4: 240-250.

26. Younis A.M., Ibrahim A.R.S., Ibrahim S.M., AboulSoud K.A., Kabbash A.M. Microbial trans-formation of curcumin and evaluation of the biological activities of the isolated metabolites. Pharm. Sci. Res., 2016; 8(10): 1169-1178. 